\title{
The Impact of Virtual Team Consistency on Individual Performance and Perceptual Outcomes Over Time
}

\author{
Robert M. Fuller \\ University of Tennessee, USA \\ rfuller2@utk.edu
}

\author{
Jama D. Summers \\ University of Tennessee, USA \\ jsumme21@utk.edu
}

\begin{abstract}
This research examines how the provision of virtual team membership consistency may impact perceptions of the communication technology and interactions as well as performance. The results from a repeated measures experiment finds that virtual teams with expectations of inconsistency in membership have a more negative perception of the supporting technology, and perceive less coordination than consistent teams. Additionally, members on consistent teams perceive less interpersonal conflict, greater coordination, and enjoy greater performance outcomes. Virtual team consistency is an important construct that can provide insights to virtual team member concerns regarding team turnover and loss of social capital due to turnover. Given the ephemeral nature of virtual team membership, consistency may be a key construct for consideration in overcoming delay in virtual team engagement and social identity development.
\end{abstract}

\section{Introduction}

Virtual teams are groups of dispersed individuals who rely on communication and information technologies to interact [1]. These teams exist solely because of the technologies that support their interactions. However, they are an interesting organizational form as they allow organizations to connect disparate experts and key employees without the related costs in travel and disruptions to business. Much research has examined the nature of virtual teams, with a major interest in improving virtual team interactions and subsequent performance. Virtual teams are somewhat unique in that the very technologies that support their existence are often the same source of problems and concern [2]. Given the almost exclusive need to communicate via computermediated methods, virtual teams have several unique characteristics that influence the degree to which they can be productive and successful, and even supersede face to face teams.

A major inhibitor or facilitator of virtual team success in interactions is the development of shared identity which helps in the creation of common language, expectations, and processes by which virtual teams can improve productivity and performance. While this research is not about social identity development per se, it does recognize that the activities referenced herein are similar to those relevant to social identity development for a virtual team. The ability of virtual teams to not only develop common bases of knowledge but to actively engage them and use them in the virtual team context is important for performance. The development of these common bases for interaction is important $[3,4]$ and a common theme for virtual team performance. As a result, any disruption to this development, such as turnover or inconsistent virtual team membership, can be detrimental to interactions, perceptions, and performance.

This research attempts to better understand the influence of inconsistency in virtual team membership, that is, the degree to which membership within the team is expected to change, and its effect on various factors that influence virtual team outcomes. Pulling from literature regarding the impacts of team turnover, i.e., team member departures, and using theory on virtual team interaction, the question it asks is how does team member consistency affect key factors in virtual team member perceptions and performance? Through the use of a lab experiment with 201 virtual teams with two levels of virtual team membership consistency we find that inconsistency influences outcomes by changing interaction styles for managing conflict, by changing perceptions of technology that supports virtual team interactions, and ultimately by dampening virtual team member performance. The findings have ramifications for research on virtual team turnover and membership consistency as well as for practice.

The research continues with a brief review of the literature on team turnover in general and virtual team 
turnover specifically. This is followed by the development of hypotheses designed to test the implications of virtual team inconsistency on certain outcomes relevant to virtual teams. We next describe our experimental research design and data collection, and provide a report on the analysis performed to address the research question. We conclude with a discussion of results and ramifications of these results for research and practice.

\section{Literature Review and Hypothesis Development}

\subsection{Virtual Team Consistency}

To understand the importance of virtual team membership consistency it is relevant to consider the impact of membership consistency in physically present teams. Prior literature in management and organizational behavior has attempted to address concerns regarding and implications of team turnover, considering working teams all the way to top management teams (TMT) that govern organizations [5]. A major concern with team turnover comes from its implications for team (and even organizational) performance [6]. While much of this research centers on why individuals leave organizations, our interest is in the ramifications for interactions, perceptions, and performance for virtual teams that are subject to team member turnover.

Borrowing from the literature on individual and team turnover, we define virtual team turnover as the voluntary or involuntary departure of a team member or members from the virtual team, followed by their subsequent replacement by new virtual team members (new to the team) [6]. A major impact from turnover and the ensuing inconsistency in team membership is the loss of human and social capital which with ensuing effects on relational development, individual interactions, and behaviors that support the healthy exchange of ideas $[3,5,7]$. Two theoretical perspectives provide the basis for this research, human capital theory and social capital theory. Human capital theory suggests that the stock of human knowledge, skills, and abilities combine (in teams) to improve productivity and performance [8]. Social capital theory suggests that productivity and performance are a result of the leverage of social relationships between individuals (on teams) [9]. When teams work together, performance is positively influenced by the application of both human and social capital as shown in the use of team member knowledge and skills and the leveraging of relational trust and collaborative effort. Turnover served to diminish both bases of performance by disrupting what teams know about the human capital available for tasks, and by diminishing trust and understanding about how to work together [5].

Teams with new members likely have not developed norms for interaction and team members within would have a less defined, if any, social identity within the team [10]. Given different initial perceptions about the nature of the team, the importance of its work and even differences in the manner best used to approach the tasks at hand, it is likely that these different perceptions will be associated with greater levels of conflict [11]. While some conflict is not inherently bad, the concern is that conflict may lead to inefficient work practices, representing divergence in the approach to work, leading to less effective results.

The complexity of virtual interaction compounds this problem in virtual teams, lengthening the process by which human and social capital, influencing norms of interaction and shared mental models regarding who know what, are developed [4]. The greater the differences between virtual team members, the greater the likelihood that there will be conflict among team members [12]. Unfortunately, the development of similar mental models in a team takes time as team members interact and slowly learn and understand group identity and the working structure of the group

A second major impact identified from team turnover comes about as new members on a team do not share in the existing team social capital, and therefore have different perceptions of team interaction methods and the appropriate way to interact. Unlike face-to-face teams, virtual teams rely solely on the use of technology as an intermediary for interaction. As teams develop, they undergo various changes in the use of technology to meet interaction requirements. Both Media Synchronicity Theory (MST) [13] and Time, Interaction and Performance (TIP) theory [14] show how communication technology needs change over the life of a team as the team develops. The need for particular types of interaction styles grows or diminishes as the team develops shortcuts for communication and can rely on pre-shared codes and known cues to express meaning. Similarly, channel expansion theory suggests that perceptions of a suitable technology for communication changes as individuals develop experience communicating with the technology and with others using the technology [15]. The implications for team turnover are significant. A team with a knowledge base of how to interact with technology will have expectations for the acceptable use of that technology to support information exchange in the team. With turnover, new virtual team members attempt to understand how communication technologies are applied, or simply use their own approach to communication technology. The difference 
in understanding between existing and new team members leads to differences in what may be considered best practice in terms of communication technology, as well as differences in perceptions on the manner of use. Eventually, enough information has passed to the "new" team member for them to recognize appropriate use of the communication technology. In the meantime, however, technology use is potentially uniquely ascribed to by the team member, leading to divergent perspectives on the use of communication technology.

Finally, across both face-to-face and virtual teams, the concern regarding perceptions and acceptable interaction styles associated with team turnover and the resulting inconsistency in team membership is its ultimate impact on factors that are antecedents to performance and performance itself. As previously noted, when team membership is volatile and inconsistent, even with replacement of lost team members, the team experiences a drop in human and social capital. The associated drop in team social identity and context has a negative impact on team know-how to perform certain tasks and to efficiently exchange information [6]. The loss of human and social capital results in a loss of coordination and control knowledge, such that members on teams with these personnel changes have to re-learn the various sources and recipients of team information [16]. Team knowledge about the most efficient ways to address problems changes, due to membership changes, as expert sources must be re-determined. As major contributing behaviors to performance, participation and coordination of knowledge and activities are all impacted, leading to reductions in performance, or at least limitations in the growth of performance [17].

Participation is a key attribute leading to satisfaction and performance on virtual teams. It reflects the manner in which virtual team members share knowledge and information, and contribute to virtual team outcomes. It is through this sharing or participation that social identity in a virtual team is developed [4]. Social identity is important as it reflects common beliefs and consistency in approaches to work, interaction, and decision-making, key to the team's social capital. Particularly for group social identity, it indicates a level of understanding about group membership and expectations for interactions and exchange in the group. To the degree that a virtual team is younger in its development, it reflects greater heterogeneity of thought and expectations, leading to divergent behaviors until a definable social identity and rules of engagement are defined, and social capital is rebuilt. Prior research has shown that greater degrees of heterogeneity of membership on a virtual team leads to less quality participation by virtual team members [12].

\subsection{Hypothesis Development}

Based upon the similarities between virtual and face to face teams, turnover in virtual teams, or inconsistency in virtual team membership, would be expected to have many of the same concerns that exist in physical teams [6]. However, given the differences in the online environment and the challenges and extended time required to develop common bases of human and social capital, and team norms for appropriate interaction [18], these challenges are potentially exacerbated in the virtual online environment. Given a context where individuals have disparate expectations for how and when to communicate various information, the lack of a common understanding about communication norms should be related to difficulties and miscommunications or misunderstandings. In short, virtual teams with experienced communicators should have a very different communication experience than teams with new members and a divergent understanding of team norms and as a result, have very different perspective of each other. Prior research supports the notion that individuals on virtual teams with different expectations about interaction have significantly different perspectives of one another [19]. These different perspectives have been shown to lead to different approaches to conflict management [20] and perceptions about the appropriate use of technology [21]. As a result we expect that teams with no team member turnover or higher consistency should have greater human and social capital resulting in better interaction (conflict management) processes, and higher perceptions regarding the fit of the technology to their task [7]. As a result, teams with better interaction processes and similar perceptions of technology use should ultimately experience higher performance across different work cases. Additionally, it is anticipated that given consistent virtual team membership, these measures should all improve as the team continues to exchange and develop and establish norms of interaction. Over time the manner of interaction becomes more of a polishing action, where prior rules are re-engaged in subsequent interactions, formalizing team communication norms [22]. As a result, we hypothesize that:

H1: Given team consistency, perceptions of a)
interpersonal conflict and b) conflict management
will improve from case to case. 
H2: Given team consistency, perceptions of a) communication and b) information fit will improve from case to case.

H3: Given team consistency, perception of a) participation, b) coordination, and c) performance will improve from case to case.

Alternatively, we expect the opposite for teams with changes in virtual team members, or a lack of consistency in the virtual team membership. The ongoing change in membership will diminish the ability of team members to learn and apply interaction norms, resulting in a state of constant relearning. This relearning interferes with ongoing virtual team activities, resulting in lower levels of controlled communication, higher levels of conflict (as rules are worked out), divergence in perceptions of communication tools, and ultimately poor performance $[6,7,22]$. With continuing changes in membership, virtual teams will be in a state of flux, with unlikely gains in performance or perceptions across different work cases. As a result, for inconsistent membership virtual teams we hypothesize that:

H4: Given team inconsistency, perceptions of a) interpersonal conflict and b) conflict management will worsen from case to case.

H5: Given team inconsistency, perceptions of a) communication and b) information fit will worsen from case to case.

H6: Given team inconsistency, perception of participation, coordination, and performance will worsen from case to case.

\section{Research Method}

This research utilized a laboratory experiment with a $1 \times 2$ repeated measures factorial design. The between subjects factor, team consistency, had two levels indicating whether participants were assigned to teams that would maintain the same or different team membership throughout the study. Participants on teams performed two cases over the course of the study and constructs were measured twice. Therefore, a within-subjects factor, case, was used to represent the repeated measures over the two case exercises.

\subsection{Participants}

Participants in the research were drawn from a large undergraduate productivity software course at a large public university in the southeastern United States. Participants ranged in age from 18 to 41 years of age, with a mean age of 20.7 years. Participants were $62.7 \%$ male with a mean of 2.7 years of work experience, and most with no experience working on a virtual team (3.9\%). A total of 803 students were recruited to participate in the research. The participants came from four sections of the course. Given the even distribution of students across the four sections of the course, 201 teams were created using one student from each of the four sections resulting in all teams consisting of four members (except one 3-person team). Students were randomly assigned to a team and each team was associated with one of the team member consistency treatments.

\subsection{Task}

The two cases performed by the teams were both spreadsheet development tasks. In both cases, teams were provided with a dataset and a list of requirements for the spreadsheet. While the spreadsheet application was the same across both cases, the requirements of the deliverable differed and required the input of all team members for completion. The task is similar to that used in other research examining virtual team performance over time with various contextual conditions [23]. Teams were allotted two weeks to complete each spreadsheet development task. In total, participants worked in virtual teams on two cases, with each case taking two weeks for completion, for a total of four weeks of virtual team interaction. There was a three-week gap between cases. While deliverables for the cases were provided to the participants, other information such as how to interact or how to structure the task was not provided.

In performing the case, as this task was for a grade in the course, participants were not allowed to exchange information with one another on how to perform the task or how to communicate and structure the task. To assess the deliverables, the instructor of the course created a scoring rubric for each case and instructed graduate teaching assistants in how to score the various components of the case deliverable. To assure consistent assessment of the deliverables, the graduate teaching assistants cross-graded each other's deliverables.

\subsection{Experimental Procedures}

Participants in the research were randomly assigned to teams. The random assignment to teams was performed at the course section level, where each participant in the course section was randomly assigned to a different team. This random assignment occurred similarly across all four sections. As a result, each team was made up of participants from different 
course sections in an attempt to separate participants from friends and more likely create virtual teams where participants had no prior knowledge of each other.

Participants in each virtual team were instructed to solely communicate and exchange files using an online discussion board and file exchange tool. For this we created 201 team workspaces using Microsoft SharePoint. Teams had the ability to create threads, create messages, respond or reply to prior messages, and post files to share to team members. Examination of the communication between team members did not provide any obvious evidence of teams meeting together off-line or breaking rules. While some teams did share mobile telephone numbers, based on the communications captured in the team workspace there is no evidence that these external tools were used. To improve the interaction experience, the discussion board on SharePoint was set to its lowest setting for message refresh, so participants could immediately see posts from team members. Assessment of the experienced refresh time suggests that the discussion board refreshed continuously every 15 seconds at the longest.

After participants were assigned to teams and completed informed consent to allow the researchers access to their interaction and survey data, a brief overview of the SharePoint workspace was provided, method of access was presented, and students were informed of the constraints on interaction outside of the workspace. Before beginning each case, participants were asked to fill out a survey to capture demographic information and initial attitudes towards team work and software. Upon completion of each case, participants completed a second survey to capture perceptions of the interactions, other team members, and their experience in the virtual team. In addition, individual scores were collected for each case upon completion.

As the two cases were a course requirement, no additional incentives or benefits were provided to the students for their participation in the study. However, to successfully complete the cases, the participants had to make use of the team workspace which was also a course requirement.

\subsection{Variables}

The independent variable for this research is virtual team (VT) consistency. The dependent variables of interest in this research are Communication fit, Information fit, Participation, Coordination, Interpersonal Conflict, Conflict management, and Performance. These constructs were all measured using 7 point-Likert scales ranging from 1 (strongly disagree) to 7 (strongly agree). A factor analysis was conducted to test for reliability, Cronbach's alpha is reported for each construct below. A copy of the measurement instrument is available from the authors. Descriptive statistics for the dependent variables by treatment and case are presented in Table 1.

VT consistency is the degree to which the membership of a virtual team is expected to not change. This definition is in contrast to other research that has looked at group instability or volatility and its influence on organizational or team outcomes [6]. Instability indicates the proportion of individuals who leave the group over a given time period [24]. In this research, we approach consistency as the opposite, the number of people who remain in the group (thereby maintaining social capital). To maximize the difference in our treatment, we have two levels of consistency, either completely consistent or completely inconsistent where all team members are replaced.

Communication fit is the degree to which the communication support provided by team software is perceived to match the manner in which team members wish to communicate [22]. This construct was measured using eight items adapted from [22] (alpha $=$ 0.879). Items included statements such as: I could easily express my ideas to others through the technology and Using the technology, the team could effectively communicate their ideas.

Information fit is the degree to which the individuals perceive the team software as providing support to aggregate, sort, manage, or otherwise organize the information needed and used to perform the team task [22]. This construct was measured using five items adapted from [22] (alpha $=0.889$ ). Sample items included: The technology displayed information in a readable format, and I found the technology useful in how it presented the information for working on the project.

Participation is the perception by team members regarding their ability to contribute to the group by communicating and exchanging information in the virtual team [12]. This construct was measured using five items adapted from [12] (alpha $=0.897)$, such as: $I$ always felt free to voice my comments during the meeting and Team members responded to the comments made by others. Coordination is the degree to which team members perceive that they can work together by being able to determine who has what key information and how that information can be stored and accessed [25]. This construct was measured using five items adapted from [25] (alpha $=0.897$ ). Sample items included: Team members can get the information they need from other members in a timely fashion and We can align our collective knowledge and efforts with task demands. 
Interpersonal conflict is defined as conflict arising from interpersonal differences and incompatibilities when working together [11]. This construct was measured using six items adapted from [20] (alpha = 0.952), such as: Personality conflicts are evident in the team and People take arguments in the team personally.

Conflict management is the degree to which team members perceive an approach to conflict that is preferred and can bring about resolution on the team [12]. This construct was measured using six items adapted from [12] (alpha $=0.922)$. Items included statements such as: I tried to investigate an issue with my team members to solve a problem together and I exchanged accurate information with teammates to solve a problem together.

Performance is the participants' individual contribution and performance on the individual aspects of the team case. It consists of the scores received on separately submitted assignments and the quiz associated with the case deliverables.

\section{Table 1. DV Means (Std Dev)}

\begin{tabular}{lcccc} 
& \multicolumn{2}{c}{ Case 1 } & \multicolumn{2}{c}{ Case 2 } \\
\cline { 2 - 5 } Item & Consist & $\begin{array}{c}\text { Not } \\
\text { Consist }\end{array}$ & Consist & $\begin{array}{c}\text { Not } \\
\text { Consist }\end{array}$ \\
\hline Comm & 3.95 & 4.13 & 3.90 & 3.77 \\
Fit & $(1.19)$ & $(1.18)$ & $(1.14)$ & $(1.16)$ \\
\hline \multirow{2}{*}{ InfoFit } & 4.06 & 4.16 & 3.85 & 3.66 \\
& $(1.21)$ & $(1.12)$ & $(1.27)$ & $(1.24)$ \\
\hline \multirow{2}{*}{ Partic } & 5.32 & 5.44 & 5.49 & 5.42 \\
& $(1.05)$ & $(1.08)$ & $(1.12)$ & $(1.12)$ \\
\hline \multirow{2}{*}{ Coord } & 4.50 & 4.54 & 4.39 & 4.33 \\
& $(1.25)$ & $(1.21)$ & $(1.39)$ & $(1.38)$ \\
\hline Intp & 2.61 & 2.45 & 2.23 & 2.33 \\
Confl & $(1.37)$ & $(1.29)$ & $(1.24)$ & $(1.28)$ \\
\hline Confl & 5.32 & 5.42 & 5.46 & 5.49 \\
Mgt & $(1.05)$ & $(1.01)$ & $(1.09)$ & $(1.05)$ \\
\hline \multirow{2}{*}{ Perf } & 28.95 & 30.00 & 32.34 & 31.71 \\
& $(5.53)$ & $(4.58)$ & $(6.23)$ & $(6.84)$ \\
\hline
\end{tabular}

To ensure adequate discriminant validity of our measures, a confirmatory factor analysis (CFA) was performed. The results of the CFA indicate that each measure loaded properly on its related construct with minimal loading on the other constructs measured. Similarly, the dependent variables were examined for normality and equality of error variances. The kurtosis and skew values for the dependent variables were all below 1, providing support that the data were normally distributed. Tests of homogeneity of covariance matrices were also tested with Box's M suggesting that the matrices were dissimilar $(p=0.008)$. However, given Box's sensitivity to large sample sizes, we also examined Levene's test and find that none of our variables exhibit differences in error variance suggesting we have homogeneity of error variances. In addition, repeated measures MANCOVA is robust to violations of homogeneity of covariance matrices, therefore believe our results to still be valid.

\section{Results}

The data were analyzed using SPSS 22. A repeated measures MANCOVA was performed, including a team variable to account for the fact that our individual measures were nested in teams [26]. The team variable (TEAM) accounts for any common variance due to a team effect on members who are on the same team. Before the data was analyzed, the results of a manipulation check were analyzed to assure that participants properly recognized the treatment to which they were assigned. Participants failing the manipulation check were dropped from the analysis (incorrectly indicating the type of team member consistency through the study). In addition, participants not fully completing the surveys or dropping the class were removed, given the nature of repeated measures analysis to require participants to have data in both cases for analysis. This left a total of 290 participants in the consistent treatment and 268 participants in the inconsistent treatment.

An initial repeated measures (RM) MANOVA was performed to assess the overall effects of consistency and case (over time) on the dependent variables. The results for the RM MANOVA are presented in Table 2a. Based on the results of the RM-MANOVA, a univariate ANOVA examining specific effects on the dependent variables was performed. Given the lack of significance for Consistency, this main effect is not represented in Table $2 b$, but the significant effects are. For brevity, the team-based effects are not provided, but are available on request. Given our interest in the main and interactive effects of our main constructs and case, these are included.

Table 2a. RM-MANOVA Results

\begin{tabular}{lccc} 
Effect & Wilks & F & Sig. \\
\hline Consist & 0.994 & 0.33 & 0.94 \\
TEAM(Consist) & 0.048 & 1.02 & 0.36 \\
Case & 0.757 & 16.40 & $\mathbf{0 . 0 0}$ \\
Case * Consist & 0.928 & 3.98 & $\mathbf{0 . 0 0}$ \\
Case * & & & \\
Team(Consist) & 0.045 & 1.04 & 0.18 \\
\hline
\end{tabular}


Table 2b. Univariate Results

\begin{tabular}{llcc} 
Effect & Measure & F & Sig. \\
\hline Case & IntpConf & 11.312 & $\mathbf{0 . 0 0}$ \\
& ConfMgt & 3.085 & $\mathbf{0 . 0 8}$ \\
& CommFit & 13.492 & $\mathbf{0 . 0 0}$ \\
& InfoFit & 36.802 & $\mathbf{0 . 0 0}$ \\
& Particip & 0.573 & 0.45 \\
& Coordin & 9.307 & $\mathbf{0 . 0 0}$ \\
& Perf & 54.693 & $\mathbf{0 . 0 0}$ \\
\hline Case * Consist & IntpConf & 4.857 & $\mathbf{0 . 0 3}$ \\
& ConfMgt & 0.372 & 0.54 \\
& CommFit & 9.851 & $\mathbf{0 . 0 0}$ \\
& InfoFit & 13.898 & $\mathbf{0 . 0 0}$ \\
& Particip & 5.449 & $\mathbf{0 . 0 2}$ \\
& Coordin & 1.979 & 0.16 \\
& Perf & 6.281 & $\mathbf{0 . 0 1}$ \\
\hline
\end{tabular}

\subsection{H1, H2, H3 Results}

Paired comparisons were performed to test the hypotheses for those univariate analyses that had significant effects. Result of paired comparison tests are shown in Table 3. Consistent with H1, participants on teams that were provided with virtual team consistency did have (H1) improved perceptions of interpersonal conflict $(\mathrm{p}=0.001)$, but conflict management was not found to be significant ( $\mathrm{p}=$ 0.083). Participants did not perceive (H2) improved communication fit nor information fit from case to case. Finally, participants did perceive (H3) improvements in participation $(\mathrm{p}=0.024)$ and higher performance $(p=0.001)$ but no improvement in coordination between cases. These results provide partial support for $\mathrm{H} 1$ and $\mathrm{H} 3$.

\subsection{H4, H5, H6 Results}

In testing teams with no virtual team consistency, participants did not perceive any difference in interpersonal conflict or in conflict management activities (H4). Participants did perceive (H5) diminished communication fit $(\mathrm{p}=0.001)$ and information fit $(p=0.001)$. Finally, participants did not perceive any change in (H6) participation however, coordination did worsen $(\mathrm{p}=0.003)$. Finally, participants did improve their performance $(p=0.001)$ from case to case. These results provide support for $\mathrm{H} 5$, but only partial support for H6.
Table 3. Pairwise Comparisons

\begin{tabular}{lccc} 
Measure & Consist & Diff & Sig. \\
\hline VT Consistency & & & \\
\hline IntpConf & YES & -0.344 & $\mathbf{0 . 0 0 0}$ \\
ConfMgt & YES & 0.122 & 0.083 \\
CommFit & YES & -0.028 & 0.695 \\
InfoFit & YES & -0.128 & 0.087 \\
Particip & YES & 0.155 & $\mathbf{0 . 0 2 4}$ \\
Coordin & YES & -0.103 & 0.228 \\
Perf & YES & 3.555 & $\mathbf{0 . 0 0 0}$ \\
\hline No VT Consistency & & \\
\hline IntpConf & NO & -0.072 & 0.429 \\
ConfMgt & NO & 0.059 & 0.434 \\
CommFit & NO & -0.354 & $\mathbf{0 . 0 0 0}$ \\
InfoFit & NO & -0.535 & $\mathbf{0 . 0 0 0}$ \\
Particip & NO & -0.079 & 0.282 \\
Coordin & NO & -0.280 & $\mathbf{0 . 0 0 3}$ \\
Perf & NO & 1.756 & $\mathbf{0 . 0 0 1}$ \\
\hline
\end{tabular}

\section{Discussion}

The results of our experiment indicate that the provision of consistency of team membership on a virtual team can have various effects on performance and perceptions of virtual team conflict, interactions, and even the efficacy or appropriateness of technology support for the team. Our results indicate that when provided with consistency in membership, virtual team members perceive lower levels of interpersonal conflict, greater participation and ultimately performance increases over the execution of two cases.

When virtual team members were not provided with assurances of virtual team consistency, perceptions of the fit of the technology in terms of both communication and information decreased, perceptions of coordination activities decreased. Interestingly, performance did increase for these virtual team members, but it was not at the same rate (less than half) as those individuals on teams with consistent team membership.

This research has several implications for theory and practice. Although much of the research on inconsistency in team membership and turnover focus on individual reasons for turnover and the performance impacts associated with such team inconsistency, this research highlights not only how team consistency can 
impact virtual team performance, but also focuses on the implications for virtual team interactions and perceptions of the team and technology.

From the perspective of TIP theory and channel expansion theory, teams with greater consistency will develop shared mental models regarding interaction styles and perceptions of technology over time. The results of this research indicate that this development may hinder perceptions of the fit of the technology for both communication and information in teams with inconsistent membership. In other words, prior expectations related to interaction styles are no longer salient with the change in membership, creating discord between heterogeneous members' expectations regarding the technology and diminishing perceptions related to technology fit.

For organizations managing virtual teams, our results highlight the relationship between consistent team membership and the development of team shared identity. Although teams with inconsistent membership did see improved performance over time, the magnitude was not as great as those in consistent teams. Likewise, inconsistent team membership diminished both team member perceptions of the efficiency of the team in terms of both coordination and technology fit.

\subsection{Limitations and Future Research}

The result of this research should be considered in light of the limitations that are inherent in laboratory experiments that include the use of student subjects. As noted by [27] and others, the type of technology applied in a virtual team context can have a significant impact on team interactions and results. The specific configuration of the technology for this research, a discussion forum that provided interactions in a more serialized, deliberate manner, could potentially drive results in a way due to the limitations for interaction available in the technology. The SharePoint workspace template applied in this research provided typical virtual team mechanisms for interaction, but other types of interaction such as synchronous chat-oriented interactions were not supported. Although only the teams with inconsistent membership seemed to be affected by the technology configuration, it is not known how teams may have performed differently given a different feature set for interaction. Future research could assess the manner in which interaction could be configured to better support the communication preferences of the participants in the virtual team. Given the limited time of interaction (two weeks), a technology with a more interactive capability might have allowed for a greater amount of social exchange to occur $[28,29]$, resulting in higher levels of team member salience and mitigating a potential lack of investment in team social identity development due to the effect of inconsistency in team membership.

The length of time for the research was two weeks (per case) which in prior research has been found to be adequate for participants to share enough social and task data to be successful in virtual team projects [23]. Our research in this area has shown that participants do convey quite a bit of information in that period of time, enough to generate opinions of team members and to develop skills in using the technology. Likewise, it is enough time for participants to be creative in their use of the technology, forming new mechanisms to improve their interaction experience in this environment. While it is unlikely that team members did not have enough time to become adept at using the technology, additional research could examine the length of time for exchange to develop social identity or to determine alternative methods for technology use to meet requirements. Some prior research suggests that online interactions can take longer for social identity to form $[4,30]$, but it also can facilitate certain types of interaction that may ultimately speed team development [19].

The development of interaction norms and team shared identity may also be influenced by the context of the workplace. The SharePoint workspace used in this experiment was accessible only for the course of the experiment whereas the virtual workspace in an organization is often longstanding. Such contexts may be subject to community norms of interaction; therefore, the speed by which team social identity is developed may be reduced given a set of standard expectations. Future research could examine team consistency in contexts where community norms are likely to have developed. Similarly, a context in which teams generally have very high or low consistency may have different baseline perceptions of what makes a team effective. Additional research may verify the effects of virtual team consistency in such an environment.

The use of student subjects has come under fire recently due to the potential inappropriateness of the use of this group to generalize to working groups that may be qualitatively different $[31,32]$. We recognize that younger online participants often have very different preferences and prior experiences working and especially communicating in a virtual environment. The task for our teams was not a concocted task for experimental purposes, but an actual design and development task for compensation (an important grade in the course). We did not provide financial incentive for participation, but participation was mandatory for class credit. While some participants still chose not to participate, a larger 
number of them did, and exhibited behaviors typical of a work environment. Clearly this research would benefit from replication in a different context, but for the purposes of understanding relevant virtual team structures that may impact perceptions, interactions and performance, the use of student participants is valid and useful given the personal importance of the task to them.

\section{Conclusion}

Prior literature and theory regarding team membership and likewise virtual team membership suggests that human and social capital in a virtual team is often hard to identify and takes time for teams to develop transactive memory systems to understand the contributions that team members can make. When virtual team membership is fluid, like regular teams, the changes in membership due to member turnover can have important negative effects on perceptions of

\section{References}

[1]. Townsend, A.M., DeMarie, S.M., and Hendrickson, A.R. (1998) Virtual teams: Technology and workplace of the future. Academy of Management Executive. 12(3): p. 17-29.

[2]. Brown, S.A., Fuller, R.M., and Vician, C. (2004) Who's afraid of the virtual world? Anxiety and computermediated communication. Journal of the Association for Information Systems. 5(2): p. 79-107.

[3]. Maynard, M.T. and Gilson, L.L. (2014) The role of shared mental model development in understanding virtual team effectiveness. Group \& Organization Management. 39(1): p. 3-32.

[4]. Postmes, T., Spears, R., and Lea, M. (2000) The formation of group norms in computer-mediated communication. Human Communication Research. 26(3): p. $341-371$.

[5]. Messersmith, J.G., Lee, J.Y., Guthrie, J.P., and Ji, Y.Y. (2014) Turnover at the top: Executive team departures and firm performance. Organization Science. 25(3): p. 776793

[6]. Hausknecht, J.P. and Trevor, C.O. (2011) Collective turnover at the group, unit, and organizational levels: Evidence, issues, and implications. Journal of Management. 37(1): p. 352-388.

[7]. Pee, L.G., Kankanhalli, A., Tan, G.W., and Tham, G.Z. (2014) Mitigating the impact of member turnover in information systems development projects. Ieee the team affect and performance. The results of our research indicate that virtual team consistency in membership allows teams to achieve higher potentials in their performance, and that turnover or inconsistency in virtual team membership has a dampening effect on performance and impacts various perceived aspects of the virtual team context. With inconsistency, perceptions of the appropriateness of technology is negatively affected as team members recognize and perhaps desire alternative methods of communicating to overcome communication limitations in the virtual environment. Perceptions of conflict and conflict management do not change, but coordination is seen to be less, resulting in lower relative performance to teams with consistent membership.

Transactions on Engineering Management. 61(4): p. 702716.

[8]. Becker, G. (1964) Human capital, New York, NY: Columbia University Press.

[9]. Leana, C.R. and Van Buren, H.J. (1999) Organizational social capital and employment practices. Academy of Management Review. 24(3): p. 538-555.

[10]. Reicher, S., Spears, R., and Postmes, T. (1995). A social identity model of deindividuation phenomena. In W. Strobe and M. Hewstone (Eds.), European review of social psychology (Vol. 6). New York, NY: Taylor and Francis.

[11]. Mortensen, M. and Hinds, P.J. (2001) Conflict and shared identity in geographically distributed teams. International Journal of Conflict Management. 12(3): p. 212-238.

[12]. Paul, S., Samarah, I.M., Seetharaman, P., and Mykytyn, P.P. (2004) An empirical investigation of collaborative conflict management style in group support system-based global virtual teams. Journal Of Management Information Systems. 21(3): p. 185-222.

[13]. Dennis, A.R., Fuller, R.M., and Valacich, J.S. (2008) Media, tasks, and communication processes: A theory of media synchronicity. MIS Quarterly. 32(3): p. 575-600.

[14]. McGrath, J.E. (1991) Time, interaction, and performance (tip) - a theory of groups. Small Group Research. 22(2): p. 147-174.

[15]. Carlson, J.R. and Zmud, R.W. (1999) Channel expansion theory and the experiential nature of media 
richness perceptions. Academy of Management Journal. 42(2): p. 153-170.

[16]. Lewis, K., Belliveau, M., Herndon, B., and Keller, J. (2007) Group cognition, membership change, and performance: Investigating the benefits and detriments of collective knowledge. Organizational Behavior and Human Decision Processes. 103(2): p. 159-178.

[17]. Bunderson, J.S., Van der Vegt, G.S., and Sparrowe, R.T. (2014) Status inertia and member replacement in roledifferentiated teams. Organization Science. 25(1): p. 57-72.

[18]. Graham, C.R. (2003) A model of norm development for computer-mediated teamwork. Small Group Research. 34(3): p. 322-352.

[19]. Walther, J.B. and Bunz, U. (2005) The rules of virtual groups: Trust, liking, and performance in computermediated communication. Journal of Communication. 55(4): p. $828-846$.

[20]. Hinds, P.J. and Mortensen, M. (2005) Understanding conflict in geographically distributed teams: The moderating effects of shared identity, shared context, and spontaneous communication. Organization Science. 16(3): p. 290-307.

[21]. Shih, H.P., Lai, K.H., and Cheng, T.C.E. (2015) Examining structural, perceptual, and attitudinal influences on the quality of information sharing in collaborative technology use. Information Systems Frontiers. 17(2): p. 455-470.

[22]. Fuller, R.M. and Dennis, A.R. (2009) Does fit matter? The impact of task-technology fit and appropriation on team performance in repeated tasks. Information Systems Research. 20(1): p. 2-17.

[23]. Fuller, R.M. and Harding, M. (2015). The impact of interaction anticipation and incentive type on shared leadership and performance in virtual teams. In T.X. Bui and R.H. Sprague (Eds.), 2015 48th hawaii international conference on system sciences (pp. 732-741).

[24]. Price, J.L. (1977) The study of turnover, Ames, IA: Iowa State University Press.

[25]. Maynard, M.T., Mathieu, J.E., Rapp, T.L., and Gilson, L.L. (2012) Something(s) old and something(s) new: Modeling drivers of global virtual team effectiveness. Journal of Organizational Behavior. 33(3): p. 342-365.

[26]. Walczuch, R.M. and Watson, R. (2001) Analyzing group data in mis research: Including the effect of the group. Group Decision and Negotiation. 10(1): p. 83-94.

[27]. Poole, M.S., Holmes, M., Watson, R., and DeSanctis, G. (1993) Group decision support systems and group communication - a comparison of decision-making in computer-supported and nonsupported groups. Communication Research. 20(2): p. 176-213.

[28]. Wang, Z.M., Walther, J.B., and Hancock, J.T. (2009) Social identification and interpersonal communication in computer-mediated communication: What you do versus who you are in virtual groups. Human Communication Research. 35(1): p. 59-85.

[29]. Walther, J.B. (1996) CMC: Computer-mediated communication: Impersonal, interpersonal and hyperpersonal interaction. Communication Research. 23: p. 3-43.

[30]. Spears, R., Lea, M., Corneliussen, R.A., Postmes, T., and Ter Haar, W. (2002) Computer-mediated communication as a channel for social resistance - the strategic side of side. Small Group Research. 33(5): p. 555574.

[31]. Ashton, R.H. and Kramer, S.S. (1980) Students as surrogates in behavioral accounting research - some evidence. Journal of Accounting Research. 18(1): p. 1-15.

[32]. Compeau, D., Marcolin, B., Kelley, H., and Higgins, C. (2012) Generalizability of information systems research using student subjects - a reflection on our practices and recommendations for future research. Information Systems Research. 23(4): p. 1093-1109. 